\title{
Gastric Calcifying Fibrous Tumor: A Very Rare Case Report
}

\author{
T. Vasilakaki ${ }^{\mathrm{a}}$ \\ E. Skafida ${ }^{a}$ \\ A. Tsavari ${ }^{a}$ \\ E. Arkoumani ${ }^{\mathrm{a}}$ \\ K. Koulia ${ }^{a}$ \\ D. Myoteri ${ }^{\mathrm{a}}$ \\ X. Grammatoglou ${ }^{a}$ \\ E. Moustou ${ }^{a}$ \\ N. Firfiris ${ }^{a}$ \\ D. Zisis ${ }^{b}$
}

Departments of ${ }^{\mathrm{a}}$ Pathology and ${ }^{\mathrm{b}}$ Gastroenterology, 'Tzaneion' General Hospital

of Piraeus, Piraeus, Greece

\section{Key Words}

Calcifying fibrous tumor - Stomach · Factor XIIIa - Mesenchymal tumor - Endoscopic ultrasound

\begin{abstract}
Calcifying fibrous tumor is a very rare benign mesenchymal tumor which shows a predilection for soft tissue, mesentery and peritoneum. Up to date only 7 cases have been reported in the literature confined to the gastric wall. We report a rare case of a calcifying fibrous tumor of the stomach in a 60-year-old man who presented with dyspepsia, flatulence and feeling weight. A clinical and laboratory investigation was performed with normal results. Gastroscopy revealed a bulge in the gastric body measuring $1 \mathrm{~cm}$ with normal overlying mucosa, and mucosal biopsies showed chronic gastritis. Endoscopic ultrasound of the gastric bulge showed a $1 \times 0.8 \mathrm{~cm}$ hypoechoic lesion involving the gastric wall. After the above finding a wedge resection of the stomach was performed. Microscopically the lesion consisted of well-circumscribed hypocellular hyalinized fibrosclerotic tissue with lympoplasmatic infiltrates, lymphoid aggregates and psammomatous calcifications. Lesional cells were positive for vimentin and factor XIII and negative for actin, desmin, S100p, CD117, CD34, CD31 and ALK-1. The lesion involved the muscularis propria with variable submucosal extension. Calcifying fibrous tumor has shown an excellent prognosis with recurrences being rare and showing the same morphology as the primary lesion.
\end{abstract}

\section{Indroduction}

Calcifying fibrous tumor is a very rare benign mesenchymal tumor composed of well-circumscribed hypocellular hyalinized firbrosclerotic tissue with lymphoplasmatic infiltrates, lymphoid aggregates and psammomatous or dystrophic calcifications. The 
cause and pathogenesis are unclear. The lesions were originally described in the subcutaneous and deep soft tissues mainly in children and young adults. Recently calcifying fibrous tumors have been reported in various locations such as the mesentery and peritoneum. Visceral examples usually occur in adults [1-5].

\section{Case Report}

A 60-year-old man came to our hospital complaining of dyspepsia, flatulence and feeling weight from three months ago. His past medical history included essential hypertension and hyperlipidemia. There was no family history of gastrointestinal disease. His physical examination was unremarkable. Gastroscopy was performed and revealed a bulge in the gastric body measuring $1 \mathrm{~cm}$ with normal overlying mucosa. Also there were erythema and edema of the mucosa. Mucosal biopsies showed chronic gastritis with no evidence of Helicobacter pylori. The biopsy from the bulge showed fragments of normal gastric mucosa.

Laboratory investigation including complete blood count, biochemical examination and tumor markers (CEA, Ca19-9, AFP) were normal. Endoscopic ultrasound of the gastric bulge showed a $1 \times$ $0.8 \mathrm{~cm}$ hypoechoic lesion involving the gastric wall. Within the lesion hyperechoic foci with shadowing consistent with calcifications were noted. After the above finding a wedge resection of the stomach was performed and the specimen submitted for histopathological examinations.

Microscopically the lesion consisted of well-circumscribed hypocellular hyalinized fibrosclerotic tissue with lymphoplasmatic infiltrates, lymphoid aggregates and psammomatous calcifications. The lesion involved the muscularis propria with variable submucosal extension (fig. $1, \underline{\text { fig. } 2}$, fig. 3 )

The immunohistochemical study showed that the fibroblastic spindle cells of the tumor were positive for vimentin and factor XIIIa and negative for actin, desmin, S100p, CD117, CD34, CKAE1, CKAE3, CD31 and anaplastic lymphoma kinase-1 (ALK-1) (fig. 4).

Based on the above characteristic morphologic and immunohistochemical findings a diagnosis of calcifying fibrous tumor was made.

Two years later the patient showed no evidence of recurrence.

\section{Discussion}

Calcifying fibrous tumor is a rare benign mesenchymal tumor. The cause and pathogenesis are unclear although examples have followed trauma. Tumors were reported initially in the subcutaneous and deep soft tissues mainly in children and young adults. Recently calcifying fibrous tumors have been reported in various locations such as the mesentery and peritoneum. Visceral examples usually occur in adults [1-7].

Up to date only 7 cases have been reported in the literature confined to the gastric wall. Most tumors originated in the gastric body and were incidental findings at autopsy or during surgery for other disease $[1,3,8,9]$.

Although rare, calcifying fibrous tumors must be included in the differential diagnosis from other gastric mesenchymal neoplasms such as smooth muscle tumors, schwannomas, lipomas, stromal tumors (GIST) and inflammatory myofibroblastic tumor $[1,3,5,10]$. 
The immunophenotype differs from that of other mesenchymal neoplasms because the spindle cells of the calcifying fibrous tumor show diffuse cytoplasmic staining with antibodies against factor XIIIa, CD68 and vimentin [1, 5, 11, 12].

Calcifying fibrous tumor has shown an excellent prognosis with recurrences being rare and showing the same morphology as the primary lesion.

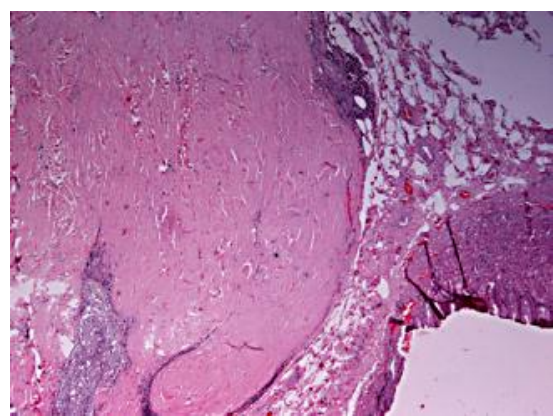

Fig. 1. Gastric calcifying fibrous tumor. H-E, $\times 100$.

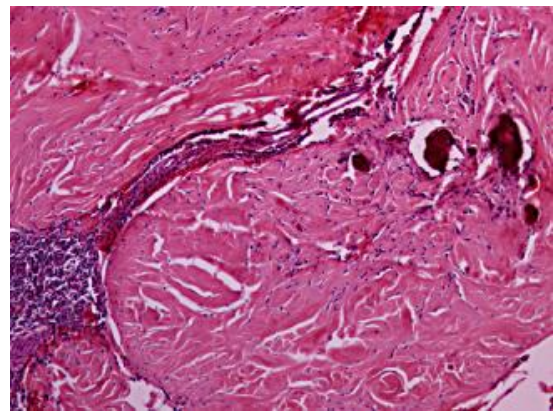

Fig. 2. Calcifying fibrous tumor. Note a lymphoid follicle. H-E, $\times 40$.

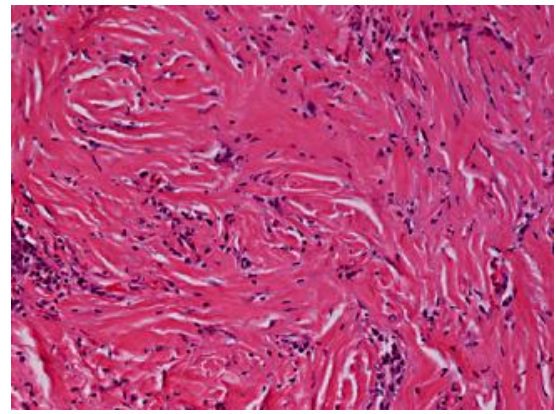

Fig. 3. Fibroblastic spindle cells of the tumor. H-E, $\times 200$. 


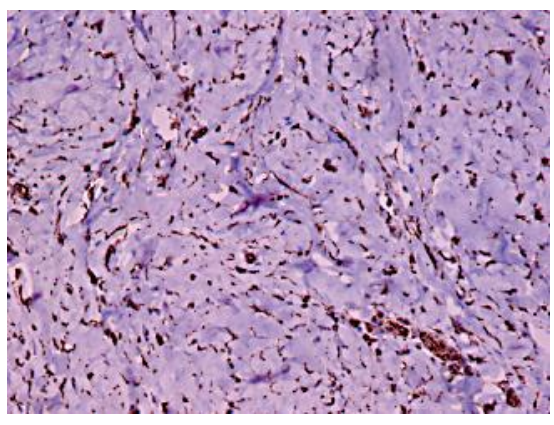

Fig. 4. Calcifying fibrous tumor. Factor XIIIa. $\times 200$.

\section{References}

1 Tan A, Dean C, Gardiner G, Ptak WT, Marron EN: Gastric calcifying fibrous tumor. Can J Gastroenterol 2006;20:487-489.

2 Rosenthal NS, Abdul-Karim FW: Childhood fibrous tumor with psammoma bodies. Clinicopathologic features in two cases. Arch Pathol Lab Med 1988;112:798-800.

-3 Agaimy A, Bihl MP, Tornillo L, Wünsch PH, Hartmann A, Michal M: Calcifying fibrous tumor of the stomach: clinicopathologic and molecular study of seven cases with literature review and reappraisal of histogenesis. Am J Surg Pathol 2010;34:271-278.

4 Jeong JE, Lee KH, Sung HJ, Cho CH: Calcifying fibrous tumor mimicking foreign body of the stomach: a case report. Korean J Pediatr Gastroenterol Nutr 2009;12:57-63.

5 Hill KA, Gonzales-Crussi F, Chou PM: Calcifying fibrous pseudotumor versus inflammatory myofibroblastic tumor: a histological and immunohistochemical comparison. Mod Pathol 2001;14:784790.

6 Kocova L, Michal M, Sulc M, Zamecnik M: Calcifying fibrous pseudotumour of visceral peritoneum. Histopathology 1997;31:182-184.

7 Weynand B, Draguet AP, Bernand P, Marbaix E, Galant C: Calcifying fibrous pseudotumour: first case report in the peritoneum with immunostaining for CD34. Histopathology 1999;34:86-87.

8 Puccio F, Solazzo M, Marciano P, Benzi F: Laparoscopic resection of calcifying fibrous pseudotumor of the gastric wall. A unique case report. Surg Endosc 2001;15:1227.

-9 Delbecque K, Legrand M, Boniver J, Lauwers GY, de Leval L: Calcifying fibrous tumour of the gastric wall. Histopathology 2004;44:399-400.

10 Sugar I, Forgacs B, Istvan G, Bogyar G, Sapy Z, Ondrejka P: Gastrointestinal stromal tumors (GIST). Hepatogastroenterology 2005;52:409-413.

11 Nickoloff BJ, Wood GS, Chu M, Beckstead JH, Griffiths CE: Disseminated dermal dendrocytomas. A new cutaneous fibrohistiocytic proliferative disorder? Am J Surg Pathol 1990;14:867-871.

12 Zamecnick M, Michal M, Boudova L, Sulc M: CD34 expression in calcifying fibrous pseudotumours. Histopathology 2000;36:183-184. 